\title{
Shedding of collagen XVII accelerates tumor growth and invasion in skin carcinogenesis
}

6000.

Célimène Galiger ${ }^{1}$, Stefanie Löffek ${ }^{1,2}$, Marc Stemmler ${ }^{3}$, Venugopal Mittapalli ${ }^{1}$, Jasmin Kröger ${ }^{1}$, Philipp Esser ${ }^{1}$, Leena Bruckner-Tuderman ${ }^{1}$, Claus-Werner Franzke CRC 850

1Department of Dermatology, Medical Center University Freiburg, Germany. ${ }^{2}$ Skin Cancer Unit of the Dermatology Department, University Hospital Essen, Germany, ${ }^{3}$ Institute of Experimental Medicine I, University of Erlangen-Nürnberg, Germany.

\section{Introduction}

Collagen XVII (CoIXVII) is an important epithelial anchorage molecule in the skin that is highly upregulated in several epithelial cancers during dysplasia-to-cancer transformation. One unique feature of collagen XVII is that its collagenous ectodomain is proteolytically released from the cell-surface.

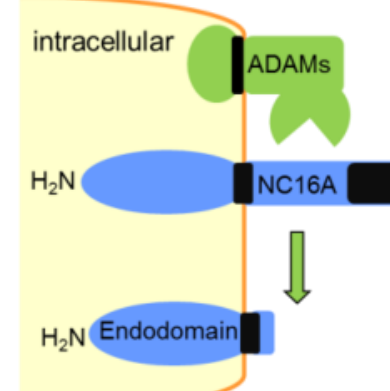

\section{0}

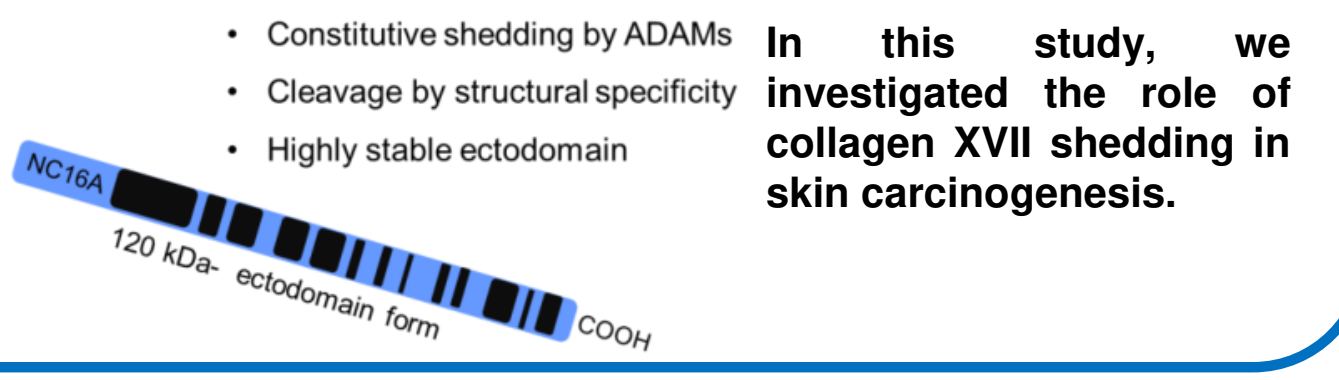

\section{Results}

Enhanced collagen XVII expression and shedding in human squamous cell carcinomas.
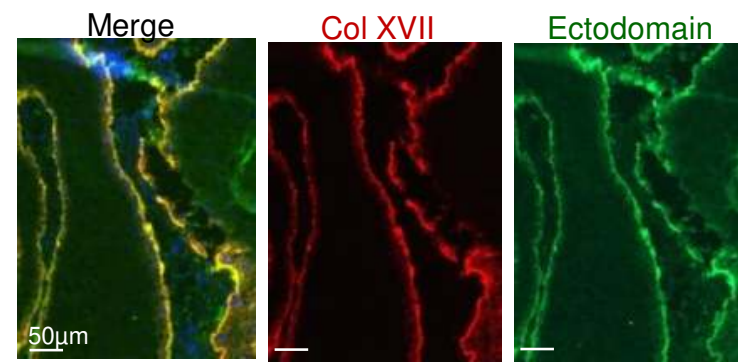

Collagen XVII and ectodoamin selective antibodies

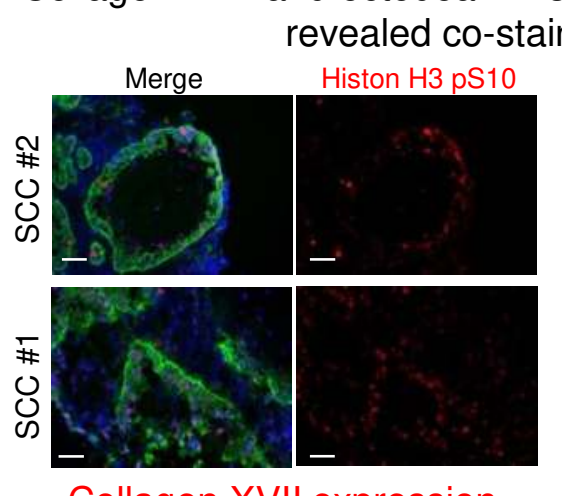

Collagen XVII expressio is linked to proliferation

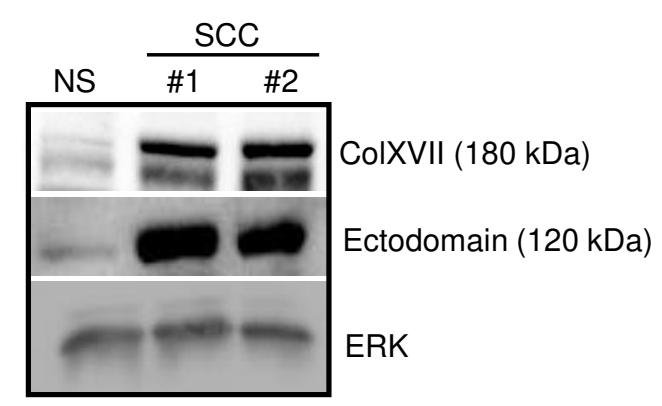

Knockdown of collagen XVII in SCC-25 cells by lentiviral shRNA causes reduced tumor growth and matrix cell invasion.

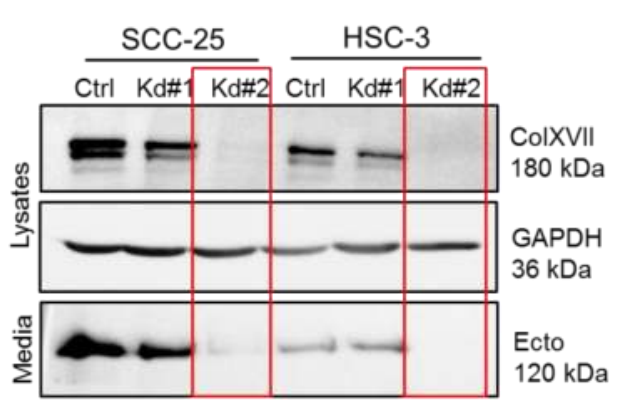

Matrigel-transmigration assay
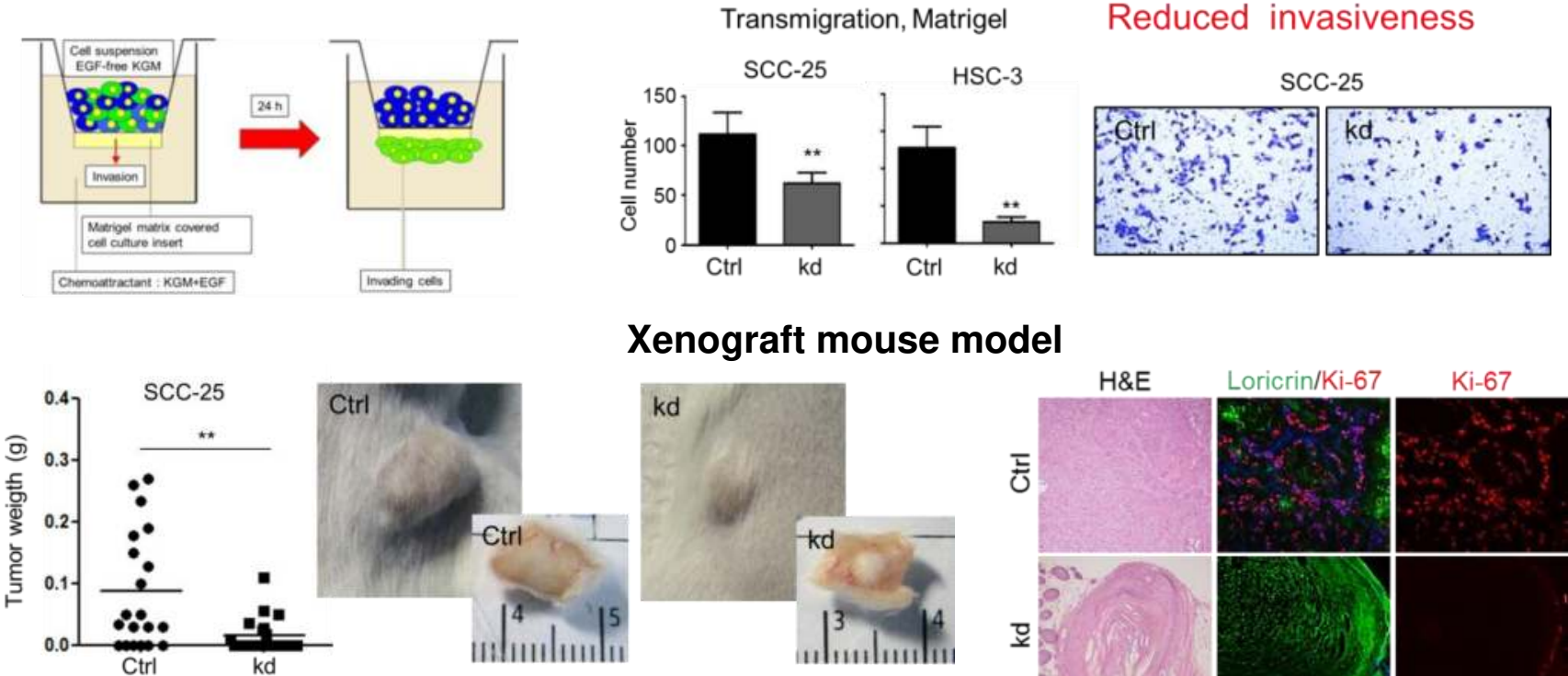

Xenograft mouse model

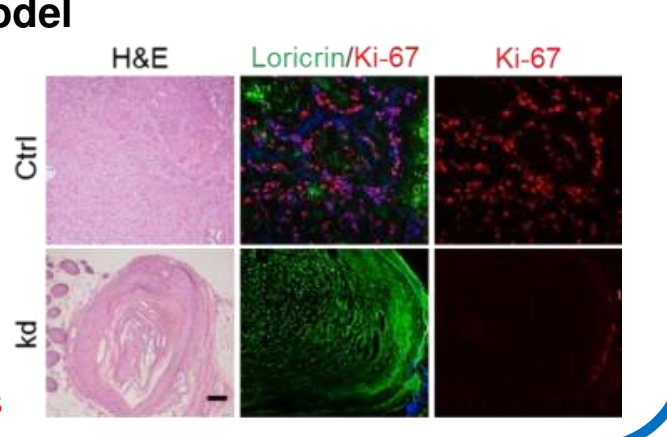

Re-expression of collagen XVII in SCC-25 Col17-kd cells rescues tumor growth and cell invasiveness.
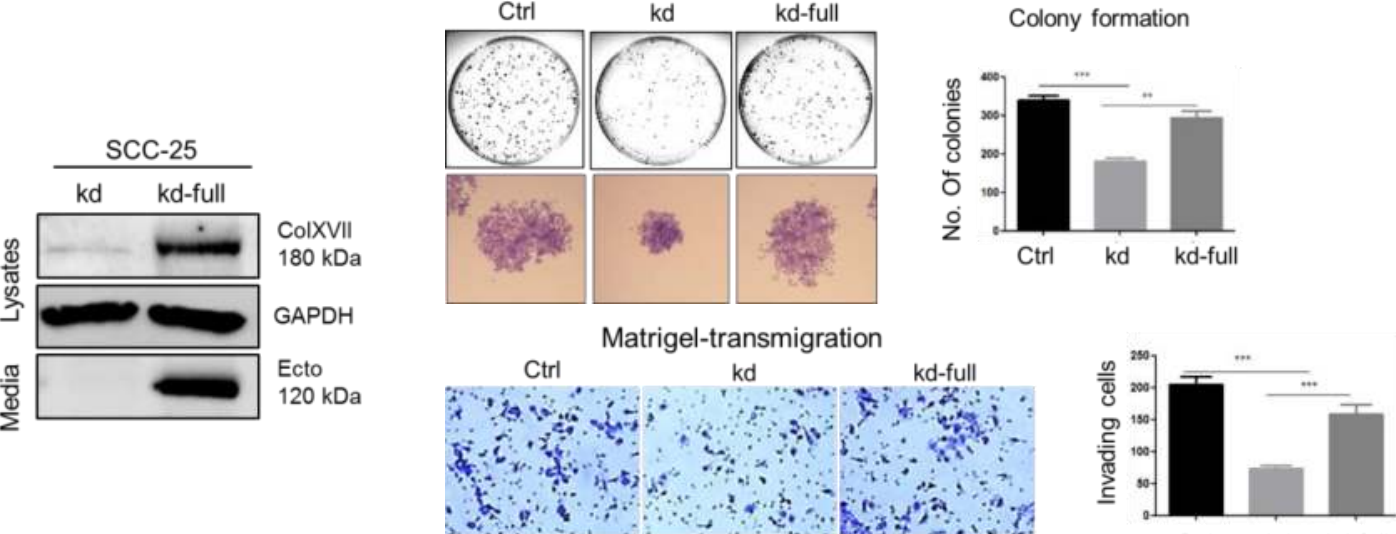

Re-expression of non-sheddable collagen XVII in SCC-25 Col17-kd cells does not restore clonogenicity and invasion.
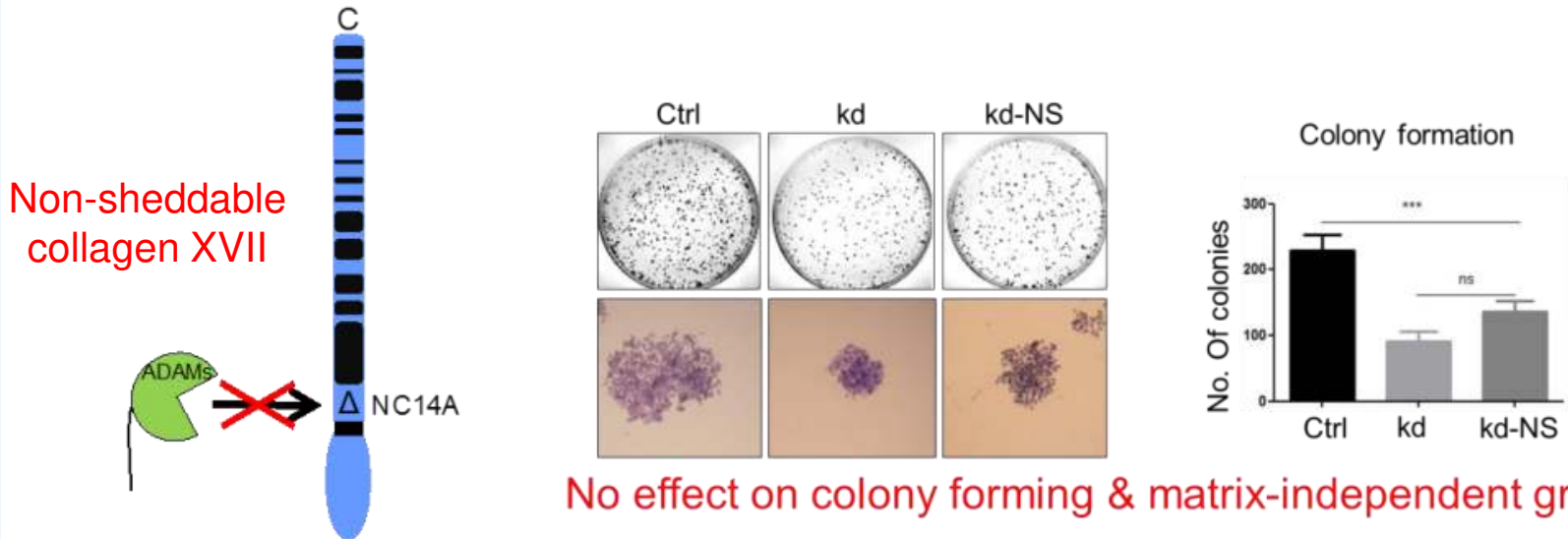

No effect on colony forming \& matrix-independent growth
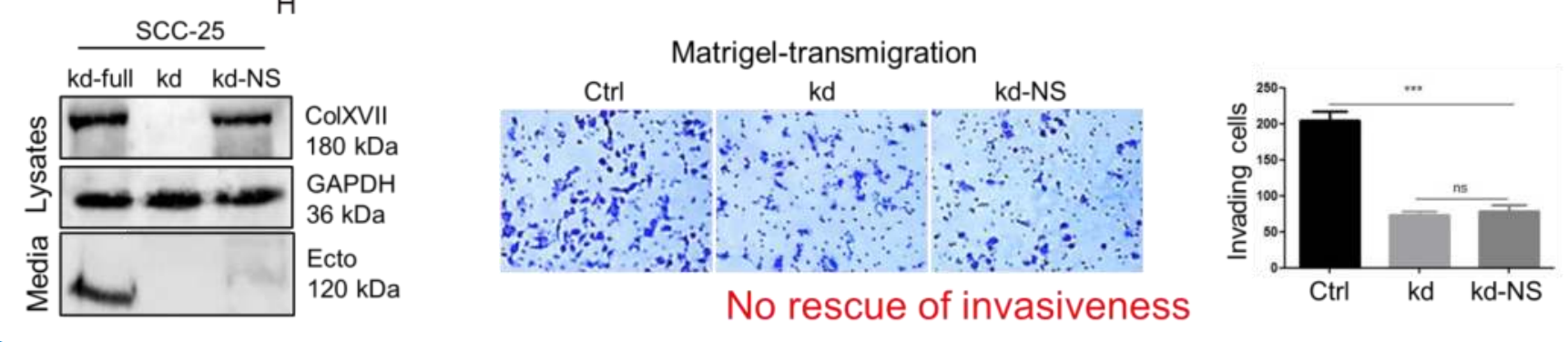

Monoclonal collagen XVII antibodies as shedding inhibitors prevent matrix-independent growth and invasion of SCC cells.
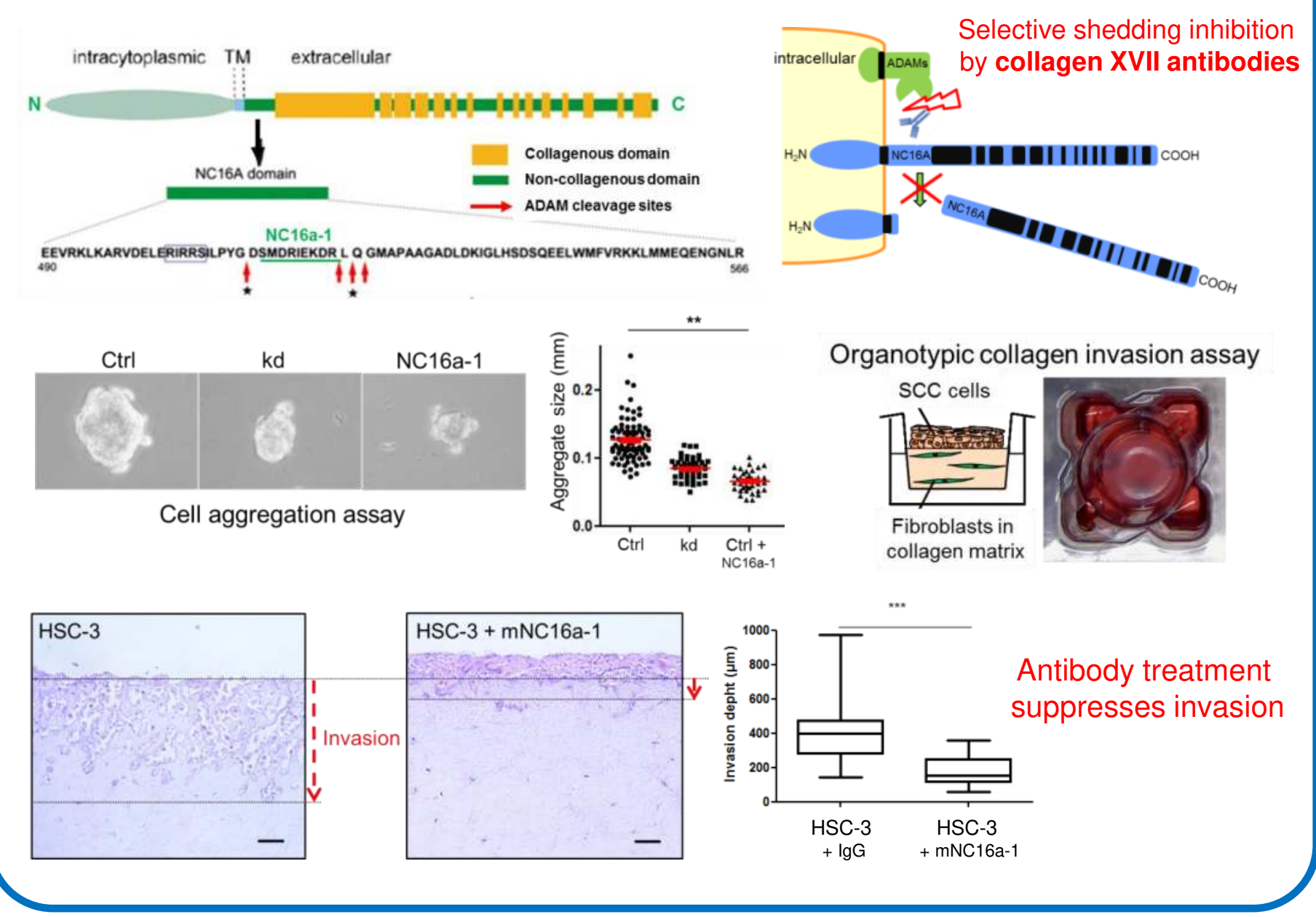

Distinct functions of collagen XVII endodomain and ectodomain.

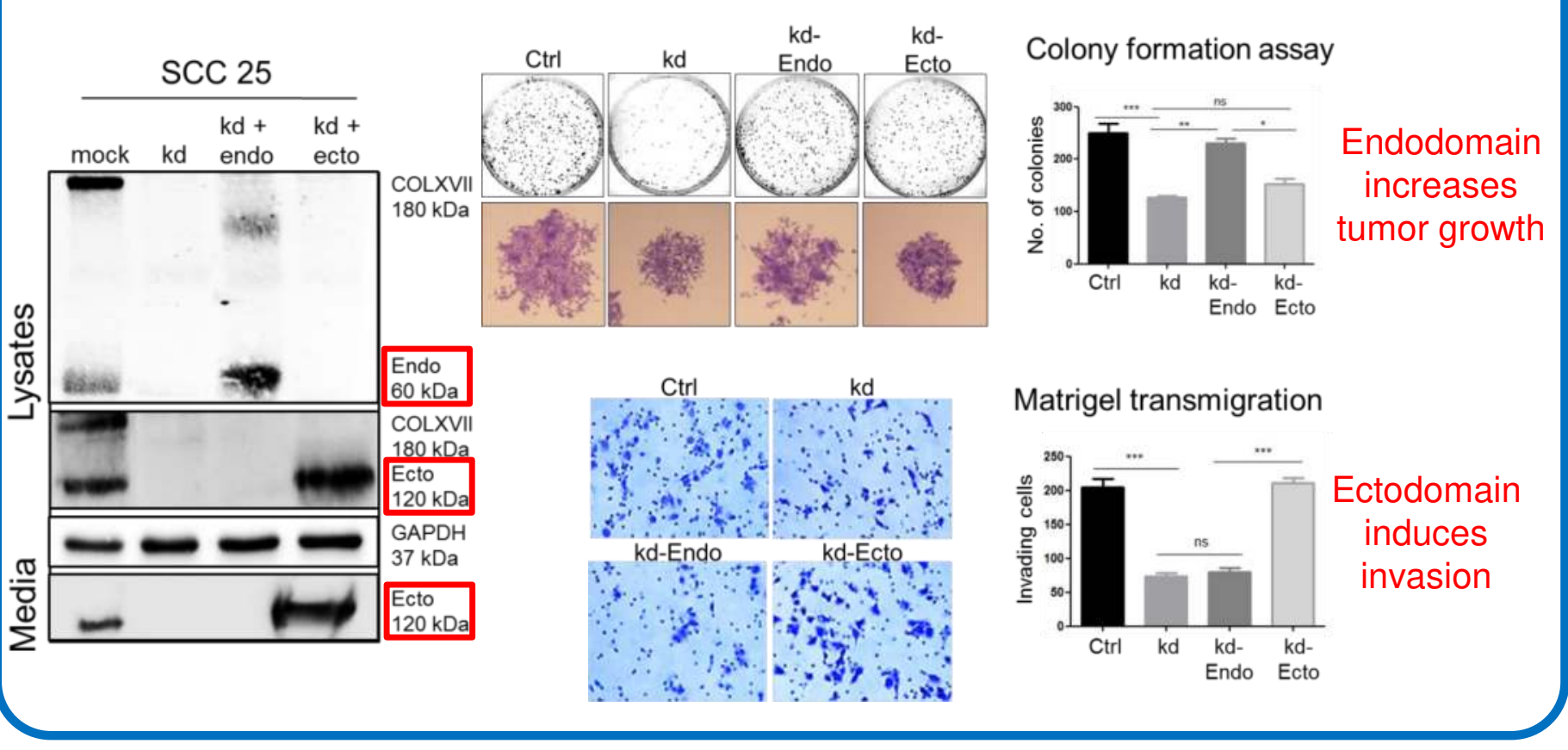

Re-expression of collagen XVII endodomain improved cell proliferation

Cell cycle analysis

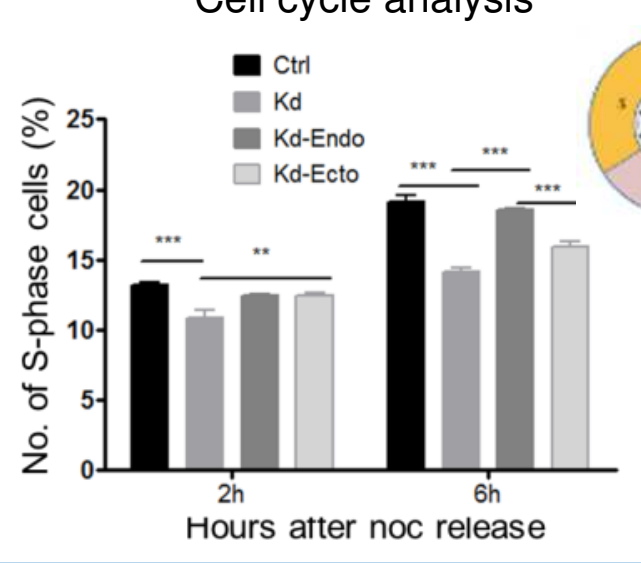

Cell proliferation (BrdU incorporation)
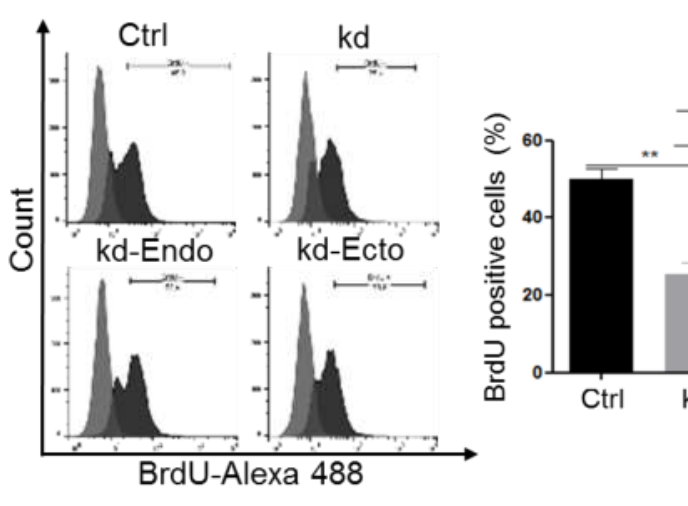

\section{Conclusion}

It seems that particularly the post-translational modification of CoIXVII by ectodomain shedding is the major driver of epithelial cancer progression. Since collagen XVII shedding is strongly increased at the invasive front of squamous cell carcinomas, local inhibition of shedding by selective blocking antibodies may offer a method to 